\title{
COVID-19 and Diabetic Ketoacidosis: A Single Center Experience
}

Balraj Singh ${ }^{1}$, Parminder Kaur ${ }^{2}$, Prem Patel ${ }^{2}$, Ro-Jay Reid ${ }^{3}$, Abhishek Kumar ${ }^{4}$, Supreet Kaur ${ }^{1}$, Nirmal Guragai $^{2}$, Abanoub Rushdy ${ }^{2}$, Mahesh Bikkina ${ }^{2}$, Fayez Shamoon ${ }^{2}$

1. Hematology/Oncology, Saint Joseph's University Medical Center, Paterson, USA 2. Cardiology, Saint Joseph's University Medical Center, Paterson, USA 3. Internal Medicine, Saint Joseph's University Medical Center, Paterson, USA 4. Hematology/Oncology, Jacobi Medical Center, New York, USA

Corresponding author: Balraj Singh, bsriar9@gmail.com

\section{Abstract}

Background and objectives: To describe the clinical characteristics and outcomes of hospitalized coronavirus disease 2019 (COVID-19) patients with diabetic ketoacidosis (DKA) -- a single center tertiary hospital experience.

Materials and Methods: A retrospective study was conducted among patients admitted to our hospital in the United States between March $1^{\text {st }}$ and June $15^{\text {th }}, 2020$ with DKA and severe acute respiratory syndromecoronavirus-2 (SARS-CoV-2) infection known as COVID-19. We compared the baseline characteristics, laboratory data, and clinical course between survivors and nonsurvivors to identify the risk factors associated with mortality in the patients with DKA.

Results: A total number of 43 patients were included in this study. The median age was 52 years. Thirtythree (76.7\%) patients were male. Median value of initial glucose on presentation was $553 \mathrm{mg} / \mathrm{dL}$ (300.0$1927.0 \mathrm{mg} / \mathrm{dL}$ ). On admission, 33 (76.7\%) patients had glycated hemoglobin (HbA1c) $\$ 8 \%(64 \mathrm{mmol} / \mathrm{mol})$ and HbA1cwas not obtained in 10 (23.3\%) patients. Acute kidney injury (AKI) was seen in 37 (86.0\%) patients, 6 (14\%) patients required renal replacement therapy and 22 (51.2\%) required mechanical ventilation. Among the 43 patients, 25 (58.1\%) died. Out of 25 patients who died 15 (60.0\%) were Hispanics, 6 (24.0\%) were White, 3 (12.0\%) were African American, 1 (4\%) was Arabic, and 1 (4\%) was Asian. The patients who died were older in age than who survived (mean age $58 \pm 6.13$ vs $46 \pm 9.39 ; p=0.023$ ). Some $95 \%$ of the patients requiring mechanical ventilation died (odds ratio [OR]: 89.25; 95\% confidence interval [CI]: 9.10874.96); $\mathrm{p}=0.001)$. Compared to survivors, nonsurvivors had significantly higher d-dimer ( $13.00 \pm 3.20$ $\mathrm{mcg} / \mathrm{mL}$ vs $6.15 \pm 3.66 \mathrm{mcg} / \mathrm{mL} ; \mathrm{p}<0.006)$ and peak ferritin values $(2763.66 \pm 1105.32 \mathrm{ng} / \mathrm{mL}$ vs $835.16 \pm$ $257.07 \mathrm{ng} / \mathrm{mL} ; \mathrm{p}=0.016$ ).

Conclusion: Our retrospective study shows COVID-19 infection may present as DKA in patients with diabetes mellitus (DM). Older age, mechanical ventilation, elevated d-dimer, and ferritin are associated with poor prognosis in these patients. Our study shows that COVID-19 is associated with substantial mortality in DKA patients and adds to the limited literature available regarding poor risk factors associated with mortality in these patients.

Review began 01/08/2021 Review ended 01/25/2021 Published 01/30/2021

\section{() Copyright 2021}

Singh et al. This is an open access article distributed under the terms of the Creative Commons Attribution License CC-BY 4.0., which permits unrestricted use, distribution, and reproduction in any medium, provided the original author and source are credited.
Categories: Endocrinology/Diabetes/Metabolism, Internal Medicine, Infectious Disease

Keywords: covid-19, severe acute respiratory syndrome-coronavirus-2, sars-cov-2 (severe acute respiratory syndrome coronavirus -2), diabetic ketoacidosis (dka), hyperglycemic hyperosmolar non-ketotic syndrome, hyperglycemic crisis, dka, type i diabetes mellitus, diabetes type 2

\section{Introduction}

The severe acute respiratory syndrome-coronavirus-2 (SARS-CoV-2), an enveloped RNA beta-coronavirus, is a novel coronavirus responsible for the current global pandemic. It has led to a global health crisis with rapidly increasing number of cases and fatalities. The spectrum of clinical manifestations of COVID-19 infection includes fever, myalgia, cough and dyspnea, and less frequently headache, diarrhea, nausea, and vomiting [1]. Most infections are not severe. According to the Chinese Center for Disease Control and Prevention, $81 \%$ patients have mild-to-moderate disease (asymptomatic or mild pneumonia), $14 \%$ have severe disease (dyspnea, hypoxia, or $>50 \%$ lung involvement on imaging within $24-48 \mathrm{~h}$ ), and only $5 \%$ have critical disease with multiple organ failure (respiratory failure, shock, or multiorgan dysfunction) [2].

Studies have shown that the rate of diabetic ketoacidosis (DKA) admissions in the United States has increased by $59 \%$ from 2003 to 2014 although the mortality has decreased from $0.51 \%$ to $0.33 \%$ in the same timeframe [3]. Hence, DKA which was once a potentially fatal disease is now well manageable. With this study, we aim to highlight the risk factors which can predict increased mortality in patients being admitted with DKA and COVID-19 infection. 


\section{Cureus}

\section{Materials And Methods}

A retrospective analysis was conducted among patients admitted to our hospital in the United States between March 1st and June 15th, 2020 with DKA and SARS-CoV-2 infection known as COVID-19. COVID19 was confirmed by real-time reverse-transcription polymerase chain reaction (PCR) assay. Diabetes was defined as glycated hemoglobin $(\mathrm{HbA} 1 \mathrm{c}) \geqslant 6.5 \%(48 \mathrm{mmol} / \mathrm{mol})$ or any established diagnosis prior admission. DKA is defined by the triad of hyperglycemia ( $>250 \mathrm{mg} / \mathrm{dL}$ ), anion gap metabolic acidosis, and ketonemia or ketonuria. The patients' electronic medical records were reviewed. Data on patients' age, sex, ethnicity, laboratory values, glycosylated hemoglobin level, oral antihyperglycemic agents (OHAs), insulin, and clinical outcomes were collected.

\section{Results}

We identified 43 patients admitted with COVID-19 and DKA from March $1^{\text {st }}$ to June $15^{\text {th }}$ of 2020 . Pertinent clinical characteristics and laboratory values are summarized in Table 1.

\section{Age (years)}

Median

IQR

40.5-64.5

Gender - no./total no. (\%)

Male

Female

10/43(23.3)

Race - no./total no. (\%)

Hispanic

$25 / 43(58.1)$

Black

9/43(20.9)

White

7/43 (16.3)

Asian

$1 / 43(2.3)$

Arabic

1/43(2.3)

BMI $\left(\mathrm{kg} / \mathrm{m}^{2}\right)$

Median

IQR

$24.2-29.9$

Diabetes history - no./total no. (\%)

Type 1 DM

7/43(16.3)

Type 2 DM

26/43(60.5)

Previously undiagnosed diabetes

10/43(23.3)

Home medications - no./total no. (\%)

Insulin

19/43(44.2)

Oral hypoglycemic agents

Metformin

21/43(48.8)

Sulfonyl urea

10/43(23.3)

SGLT2 inhibitors

$6 / 43(14.0)$

DPPIV inhibitors

4/43(9.4)

Thiazolidinediones

$1 / 43(2.3)$

GLP-1 agonist

$1 / 43(2.3)$

HbA1c on admission \% (mmol/mol)- no./total no. (\%)

$\mathrm{HbA} 1 \mathrm{c} \geq 8 \%$ ( $\geq 64 \mathrm{mmol} / \mathrm{mol})$

$33 / 43(76.7)$ 


\section{Cureus}

$\mathrm{HbA} 1 \mathrm{c}<8 \%(<64 \mathrm{mmol} / \mathrm{mol})$

0/43(0.0)

HbA1c unknown

10/43(23.3)

Initial glucose value at presentation

Median (range) - mg/L

$553.0(300.0-1927.0)$

Anion gap

Median (range) - mEq/L

25.3(12.0-45.3)

D-Dimer

Median (range) $-\mathrm{mcg} / \mathrm{mL}$

8.47(0.58-20)

Troponin

Median (range) - ng/mL

$0.1(0.01-39.0)$

CRP

Median (range) - mg/L

212.7(9.8-403)

$\mathrm{LDH}$

Median (range) - U/L

$547.0(187.0-12000)$

Ferritin

Median (range) - ng/mL

1217.0(85.0-7500.0)

Lactic acid

Median (range) - $\mathrm{mmol} / \mathrm{L}$

$3.0(0.7-10.7)$

Outcomes - no./total no. (\%)

Mechanical ventilation

22/43(51.2)

AKI

$37 / 43(86.0)$

Renal replacement therapy

$6 / 43(14.0)$

Death

25/43(58.1)

TABLE 1: Pertinent clinical characteristics, laboratory values, and outcomes in our study population.

IQR, interquartile range; BMI, body mass index; HbA1c, glycated hemoglobin; DM, diabetes mellitus; SGLT2, sodium-glucose co-transporter2; DPPIV, dipeptidyl peptidase IV inhibitors; GLP-1, glucagon-like peptide-1; AKI, acute kidney injury; CRP, C-reactive protein; LDH, lactate dehydrogenase

The median age for the patient was 52 years (inter quartile range, IQR 40.5-64.5). Thirty-three (76.7\%) patients were male. Ethnicity distribution in our patients - 25 /43 (58.1\%) were Hispanic, 9/43 (20.9\%) were African American, 7/43 (16.3 \%) were White, 1 was Asian, and 1 was Arabic. Median body mass index (BMI) was $26.7 \mathrm{~kg} / \mathrm{m} 2$. Out of 43 patients, 10 patients (23.3\%) had undiagnosed diabetes mellitus (DM), seven patients (16.3\%) had diagnosed type $1 \mathrm{DM}$ and 26 patients $(60.5 \%)$ had diagnosed type $2 \mathrm{DM}$. Amongst these 19 (44.2\%) patients who were on insulin at home, 21 (48.8\%) were on metformin, 10 (23.3\%) were taking sulfonyl urea, 6 (14\%) were on SGLT 2 inhibitors (known to increase the risk of ketoacidosis), 4 (9.3\%) were on DPPIV inhibitors, 1 (2.3\%) was on thiazolidinediones, and 1 (2.3\%) was on GLP-1 agonist. On admission, 33 patients $(76.7 \%)$ had $\mathrm{HbA} 1 \mathrm{c} \geqslant 8 \%(64 \mathrm{mmol} / \mathrm{mol})$ and none had $\mathrm{HbA} 1 \mathrm{c}<8 \%(64 \mathrm{mmol} / \mathrm{mol})$. HbA1cwas not obtained in $10(23.3 \%)$ patients.

Out of 25 patients who died -- 15 (60.0\%) were Hispanics, 6 (24.0\%) were White, 3 (12.0\%) were African American, and 1 was Arabic (4\%). Median value of initial glucose on presentation in our study population was $553 \mathrm{mg} / \mathrm{dL}$ (300.0-1927.0 mg/dL). Median value of HbA1c on presentation was $13.9 \%$ (128 mmol/mol). Some 10 patients had large ketonemia, 17 patients had moderate ketonemia, and 16 patients had small ketonemia. Acute kidney injury (AKI) was seen in 37 patients (86.0\%), 6 patients (14\%) required renal replacement therapy, and 22 patients (51.2\%) required mechanical ventilation. All the patients were started on standard treatment protocol for DKA with IV fluids and insulin infusion. 


\section{Cureus}

Table 2 shows analysis of factors associated with prognosis among patients with COVID-19 infection.

\begin{tabular}{|c|c|c|c|}
\hline Variable & Survivors & Nonsurvivors & $p$ value \\
\hline Age (years) & $46 \pm 9.39$ & $58 \pm 6.13$ & 0.023 \\
\hline BMI $\left(\mathrm{kg} / \mathrm{m}^{2}\right)$ & $26.97 \pm 4.22$ & $28.85 \pm 2.52$ & 0.4 \\
\hline HbA1c (\%) & $13.6 \pm 1.19$ & $13.37 \pm 1.32$ & 0.78 \\
\hline Glucose (mg/L) & $673.44 \pm 191.02$ & $713.58 \pm 157.05$ & 0.735 \\
\hline Anion gap (mEq/L) & $27.46 \pm 3.79$ & $25.92 \pm 3.44$ & 0.538 \\
\hline D-dimer (mcg/mL) & $6.15 \pm 3.66$ & $13.00 \pm 3.20$ & 0.006 \\
\hline Troponin (ng/mL) & $0.18 \pm 0.17$ & $3.76 \pm 3.86$ & 0.068 \\
\hline LDH (U/L) & $601.16 \pm 342.16$ & $1289.39 \pm 1023.84$ & 0.197 \\
\hline AST (U/L) & $78.72 \pm 112.26$ & $116.72 \pm 96.58$ & 0.598 \\
\hline $\operatorname{ALT}(\mathrm{U} / \mathrm{L})$ & $45.72 \pm 46.06$ & $61.08 \pm 43.89$ & 0.626 \\
\hline Lactic acid (mmol/L) & $3.07 \pm 1.06$ & $3.856 \pm 0.853$ & 0.233 \\
\hline Fibrinogen (mg/dl) & $555.66 \pm 187.90$ & $759.13 \pm 181.26$ & 0.121 \\
\hline Ferritin (ng/mL) & $835.16 \pm 257.07$ & $2763.66 \pm 1105.32$ & 0.001 \\
\hline ESR & $71.27 \pm 20.49$ & $66.48 \pm 16.17$ & 0.698 \\
\hline $\operatorname{CRP}(\mathrm{mg} / \mathrm{L})$ & $181.21 \pm 61.6$ & $229.46 \pm 36.27$ & 0.138 \\
\hline
\end{tabular}

TABLE 2: Analysis of factors associated with prognosis among patients with COVID-19 infection.

BMI, body mass index; AST, aspartate transaminase; ALT, alanine transaminase; CRP, C-reactive protein; LDH, lactate dehydrogenase; HbA1c, glycated hemoglobin; LDH, lactate dehydrogenase; ESR, erythrocyte sedimentation rate

The patients who died were older than who survived (mean age $58 \pm 6.13$ vs $46 \pm 9.39 ; p=0.023$ ). Some $95 \%$ of the patients requiring mechanical ventilation died (odds ratio [OR]: 89.25 ; $95 \%$ confidence interval [CI]: 9.103-874.9644); $\mathrm{p}=0.001$ ). Compared to survivors, nonsurvivors had significantly higher mean d-dimer $(13.00 \pm 3.20 \mathrm{mcg} / \mathrm{mL}$ vs $6.15 \pm 3.66 \mathrm{mcg} / \mathrm{mL} ; \mathrm{p}<0.006)$ and peak ferritin values $(2763.66 \pm 1105.32 \mathrm{ng} / \mathrm{mL}$ vs $835.16 \pm 257.07 \mathrm{ng} / \mathrm{mL} ; \mathrm{p}=0.016$ ). There were no statistically significant differences between the two groups (survivors vs nonsurvivors) in BMI, HbA1c, glucose, anion gap, troponin, lactate dehydrogenase (LDH), aspartate transaminase, alanine transaminase, lactic acid, fibrinogen, male sex, AKI, and renal replacement therapy (all $\mathrm{p}>0.05)$.

\section{Discussion}

In the United States, the prevalence of diabetes among adults varies with race/ethnicity and ranges from $6.8 \%$ to $15.3 \%$. Diabetes is one of the leading causes of morbidity and mortality. More healthcare resources are estimated to be spent on diabetes than on any other condition [4]. Morbidity from diabetes is a consequence of both macrovascular disease (atherosclerosis) and microvascular disease (retinopathy, nephropathy, and neuropathy) [5]. The development and progression of these complications can be delayed with management of hyperglycemia.

The care of patients with diabetes during the COVID-19 pandemic poses unique challenges. COVID-19 can precipitate severe manifestations of diabetes, includingDKA and hyperosmolar hyperglycemic state (HHS) [6]. Kamrath et al. reported a significant increase in DKA at diabetes diagnosis during the COVID-19 period in 2020 as compared to the two previous years ( $44.7 \%$ in 2020 vs $24.5 \%$ in 2019 ; vs $24.1 \%$ in 2018) [7]. Guo et al. reported higher levels of inflammation-related biomarkers in DM patients compared to non-DM patients and DM as a risk factor for the progression and prognosis of COVID-19 patients [8].

The exact pathological mechanism leading to the complication of DKA in COVID-19 is not known at present. Angiotensin-converting enzyme 2 (ACE2) serves as a functional receptor for SARS-CoV-2. ACE-2 is not only expressed in alveolar cells but also myocardial cells, proximal tubule cells of the kidney, ileum and esophagus epithelial cells, endocrine tissues of the pancreas, and bladder urothelial cells [9-10]. SARS-CoV-2 could cause direct tissue damage leading to acute hyperglycemia. 
The mortality rate in our study was much higher than that observed in the general population. Richardson et al. reported a mortality rate of $21 \%$ in a study of 5700 patients hospitalized with COVID-19 in the New York City area [11]. Another study demonstrated greater mortality in a subgroup of 24 patients with diabetes as compared to 26 patients without diabetes (16.5\% vs 0\%) [8]. Another study from New York, reported mortality of 50\% in COVID-19 patients with DKA upon admission or developed during their hospital course [12]. In our study, we observed the same trend with higher mortality (58.1\%) in COVID-19 patients with DKA. In contrary, another study showed that patients with DKA were more likely to survive compared to patients without DKA [13].

In our study, $51.2 \%$ of the patients required mechanical ventilation and $95 \%$ of these patients died (OR: 89.25; 95\% CI: 9.103-874.9644; $\mathrm{p}=0.01$ ). Previously reported study has shown mortality of $88 \%$ in COVID19 patients who were intubated [11]. However, they did not include the patients who were still alive in the ICU at the time of the study. Individuals of any age can acquire SARS-CoV-2 infection and older age has been associated with increased mortality [14-15]. In our study also, the mean age of nonsurvivors was 58 years while mean age of survivors was 46 years.

D-dimer was significantly higher on presentation in nonsurvivors vs survivors in our cohort. This is in concordance with the previous studies that have shown increased mortality in patients with elevated ddimer [15-16]. Also, ferritin has been shown to be a marker of severity and poor prognosis in COVID-19 patients [17]. In our study the patients who died had a significantly higher peak ferritin level than those who did not. Male sex, obesity, and AKI have been previously associated with increased mortality; however, in our study these were not statistically significant (all p > 0.05) [14, 18-19]. Further studies with large sample size are needed.

This study had some limitations. Firstly, our study is a single center retrospective observational study with no randomization between diabetic and nondiabetic patients and there are limitations to generalizing these findings. Secondly this study was a small number of COVID-19 patients with DKA in our sample. Thirdly, our center is a tertiary care center so most of the COVID-19 cases admitted had severe infection which could act as bias. Confounding by the use of steroids as part of COVID-19 treatment can also add to the limitations as the use of steroids can increase glucose levels. Confounding by comorbid conditions cannot be completely excluded.

\section{Conclusions}

Our retrospective study shows COVID-19 infection may present as DKA in patients with DM. Older age, mechanical ventilation, elevated d-dimer, and ferritin are associated with poor prognosis in these patients. Our study shows that COVID-19 is associated with substantial mortality in DKA patients and adds to the limited literature available regarding poor risk factors associated with mortality in these patients. Further studies with large sample size are needed to further asses the clinical characteristics, prognostic factors, and outcomes in COVID-19 patients with DKA.

\section{Additional Information \\ Disclosures}

Human subjects: Consent was obtained by all participants in this study. Animal subjects: All authors have confirmed that this study did not involve animal subjects or tissue. Conflicts of interest: In compliance with the ICMJE uniform disclosure form, all authors declare the following: Payment/services info: All authors have declared that no financial support was received from any organization for the submitted work. Financial relationships: All authors have declared that they have no financial relationships at present or within the previous three years with any organizations that might have an interest in the submitted work. Other relationships: All authors have declared that there are no other relationships or activities that could appear to have influenced the submitted work.

\section{References}

1. Guan WJ, Ni ZY, Hu Y, et al.: Clinical characteristics of coronavirus disease 2019 in China . N Engl J Med. 2020, 382:1708-1720. 10.1056/NEJMoa2002032

2. Wu Z, McGoogan JM: Characteristics of and important lessons from the coronavirus disease 2019 (COVID19) outbreak in China: summary of a report of 72314 cases from the Chinese Center for Disease Control and Prevention. JAMA. 2020, 323:1239-1242. 10.1001/jama.2020.2648

3. Desai D, Mehta D, Mathias P, Menon G, Schubart UK: Health care utilization and burden of diabetic ketoacidosis in the U.S. over the past decade: a nationwide analysis. Diabetes Care. 2018, 41:1631-1638. $10.2337 /$ dc $17-1379$

4. Dieleman JL, Baral R, Birger M, et al.: US spending on personal health care and public health, 1996-2013. JAMA. 2016, 316:2627-2646. 10.1001/jama.2016.16885

5. Papatheodorou K, Banach M, Edmonds M, Papanas N, Papazoglou D: Complications of diabetes. J Diabetes Res. 2015, 2015:189525. 10.1155/2015/189525

6. Li J, Wang X, Chen J, Zuo X, Zhang H, Deng A: COVID-19 infection may cause ketosis and ketoacidosis. Diabetes Obes Metab. 2020, 22:1935-1941. 10.1111/dom.14057

7. Kamrath C, Mönkemöller K, Biester T, et al.: Ketoacidosis in children and adolescents with newly diagnosed 
type 1 diabetes during the COVID-19 pandemic in Germany. JAMA. 2020, 324:801-804. 10.1001/jama.2020.13445

8. Guo W, Li M, Dong Y, et al.: Diabetes is a risk factor for the progression and prognosis of COVID-19 . Diabetes Metab Res Rev. 2020, e3319. 10.1002/dmrr.3319

9. Yang J-K, Lin S-S, Ji X-J, Guo L-M: Binding of SARS coronavirus to its receptor damages islets and causes acute diabetes. Acta Diabetol. 2010, 47:193-199. 10.1007/s00592-009-0109-4

10. Zou X, Chen K, Zou J, Han P, Hao J, Han Z: Single-cell RNA-seq data analysis on the receptor ACE2 expression reveals the potential risk of different human organs vulnerable to $2019-\mathrm{nCoV}$ infection. Front Med. 2020, 14:185-192. 10.1007/s11684-020-0754-0

11. Richardson S, Hirsch JS, Narasimhan M, et al.: Presenting characteristics, comorbidities, and outcomes among 5700 patients hospitalized with COVID-19 in the New York City area. JAMA. 2020, 323:2052-2059. 10.1001/jama.2020.6775

12. Chamorro-Pareja N, Parthasarathy S, Annam J, Hoffman J, Coyle C, Kishore P : Letter to the editor: unexpected high mortality in COVID-19 and diabetic ketoacidosis. Metabolism. 2020, 110:154301. 10.1016/j.metabol.2020.154301

13. Alkundi A, Mahmoud I, Musa A, Naveed S, Alshawwaf M: Clinical characteristics and outcomes of COVID19 hospitalized patients with diabetes in the United Kingdom: A retrospective single centre study. Diabetes Res Clin Pract. 2020, 165:108263. 10.1016/j.diabres.2020.108263

14. Williamson EJ, Walker AJ, Bhaskaran K, et al.: Factors associated with COVID-19-related death using OpenSAFELY. Nature. 2020, 584:430-436. 10.1038/s41586-020-2521-4

15. Zhou F, Yu T, Du R, et al.: Clinical course and risk factors for mortality of adult inpatients with COVID-19 in Wuhan, China: a retrospective cohort study. Lancet. 2020, 395:1054-1062. 10.1016/S01406736(20)30566-3

16. Zhang L, Yan X, Fan Q, et al.: D-dimer levels on admission to predict in-hospital mortality in patients with Covid-19. J Thromb Haemost. 2020, 18:1324-1329. 10.1111/jth.14859

17. Yan Y, Yang Y, Wang F, et al.: Clinical characteristics and outcomes of patients with severe covid-19 with diabetes. BMJ Open Diabetes Res Care. 2020, 8:e001343. 10.1136/bmjdrc-2020-001343

18. Tartof SY, Qian L, Hong V, et al.: Obesity and mortality among patients diagnosed with COVID- 19: results from an integrated health care organization. Ann Intern Med. 2020, 173:773-781. 10.7326/m20-3742

19. Xia P, Wen Y, Duan Y, et al.: Clinicopathological features and outcomes of acute kidney injury in critically ill COVID-19 with prolonged disease course: a retrospective cohort. J Am Soc Nephrol. 2020, 31:2205-2221. 10.1681/asn.2020040426 